\title{
Virulence and molecular characterization of Costa Rican isolates of Rhizoctonia solani from common bean
}

\author{
Floribeth Mora-Umaña ${ }^{1,2^{*}}$, Natalia Barboza ${ }^{2 *}$, Ricardo Alvarado ${ }^{3}$, Marcela Vásquez ${ }^{2}$, Graciela Godoy- \\ Lutz $^{4}$, James R. Steadman ${ }^{4} \&$ Pilar Ramírez ${ }^{2,5}$
}

${ }^{1}$ Servicio Fitosanitario del Estado (SFE), Sabana Sur, 1521-1200 San José, Costa Rica; ${ }^{2}$ CIBCM, Universidad de Costa Rica (UCR), Ciudad de la Investigación, 11501-2060, San José, Costa Rica; ${ }^{3}$ Escuela de Estadística, UCR, San Pedro, 115012060, San José, Costa Rica; ${ }^{4}$ University of Nebraska-Lincoln, 406 Plant Sciences Hall, Lincoln, NE 68583-0722, USA; ${ }^{5}$ Escuela de Biología, UCR, 11501-2060, San José, Costa Rica

Author for correspondence: Natalia Barboza, e-mail: natalia.barboza@ucr.ac.cr

\begin{abstract}
Web blight is one of the main diseases that affects bean (Phaseolus vulgaris) cultivation. It infects diverse organs at any growth stage of the plant and can be present at different altitudes in a humid tropical climate. The causal agent of this disease is Thanatephorus cucumeris in its sexual stage and Rhizoctonia solani in the anamorph. The objective of this investigation was to characterize molecular isolates of $R$. solani obtained from bean plants from diverse production regions in Costa Rica and determine their virulence. Fifty-one samples of symptomatic bean plants were collected using a global positioning system. Virulence was evaluated using the detached leaf technique. Isolates were identified using AG 1-IA, AG 1-IB, AG 1-IC, AG 1-ID, AG 2-2, AG 2-2IIIB, AG 2-2IV and AG 4 molecular markers. ITS sequences were obtained and analyzed with BLAST, aligned, and a phylogenetic tree was constructed. A high degree of virulence and genetic variability between isolates was identified and the anastomosis subgroups of isolates were independent of their geographical origin.
\end{abstract}

Key words: Phaseolus vulgaris, anastomosis group (AG), genetic variability, molecular characterization, sclerotium size, virulence.

\section{INTRODUCTION}

Common bean (Phaseolus vulgaris L.) is one of the basic components of Costa Rican and Latin-American diets. It is a valuable legume that supplies a significant amount of nutrients in developing countries (FAO, 2004; GodoyLutz et al., 2008). Web blight (WB) is a common and destructive bean disease in tropical and subtropical regions of the American continent. The causal agent is the fungus Thanatephorus cucumeris (Frank) Donk, the sexual stage of Rhizoctonia solani Kühn, a basidiomycete. It survives from one season to the next mostly via sclerotia or mycelium in crop residues in the field (Godoy-Lutz et al., 2000; Polanco et al., 1996). It is endemic to Central America and the Caribbean, and causes defoliation and severe losses in seed quality and production (Godoy-Lutz et al., 2003). Crop losses above $20 \%$ have been reported in more than 200 cultivars around the world (Beaver et al., 2002). Chemical and cultural control are the most common management techniques (Beebe et al., 1991; Godoy-Lutz et al., 1996a; Polanco et al., 1996), but during the last decades the

\footnotetext{
*These authors contributed equally to the work
}

biocontrol using different bacterial genus has been adopted as a sustainable approach for crop health management. However, they are not sufficient to avoid production losses (Solanki et al., 2013; Kanini et al., 2013).

A common bean line with high tolerance to WB in different geographical regions has not been obtained or identified (Beaver et al., 2002). Breeding efforts to create a WB resistant common bean have been limited by fungal variability in varietal reactions and the lack of knowledge of pathogen variation, mating systems and disease resistance mechanisms (Godoy-Lutz et al., 2003; Godoy-Lutz et al., 2008).

$R$. solani is a complex, heterogeneous species of several anastomosis groups (AG), which are defined as isolates with somatic or vegetative compatibility (Matsumoto, 2005). Worldwide, 14 AGs and numerous subgroups have been identified based on physical, chemical, pathogenic and molecular characteristics. Isolates can exist as multinucleate homokaryons or heterokaryons and present diverse mating strategies (homothallic or heterothallic) (González et al., 2012). Phaseolus vulgaris isolates belong mainly to AG 1 (6 subgroups), AG 2 (4 subgroups) and AG 4 (Carling, 1996; Schneider et al., 1997; Carling et al., 1999; Carling et al., 2002; Godoy-Lutz et al., 2003; GodoyLutz et al., 2008; González et al., 2006). 
When evaluated on the same plant species, virulence between AGs varies from highly virulent to non-virulent. Godoy-Lutz et al. (1996b) observed discrepancies in the field since virulence patterns of isolates from different geographical regions may vary within the same subgroup, for example AG 4 or AG 1-IE (Godoy-Lutz et al., 2000). Awareness of the pathogenicity and genetic diversity of isolates helps to explain the variation in the cultivar reaction in pathogen-host interactions (Godoy-Lutz et al., 2003).

The development and progress of the disease is favored by high temperature and relative humidity. The pathogen can be spread as rain splashed sclerotia or mycelia, often in association with plant debris, airborne basidiospores, infested soil debris and asymptomatic infected seeds (Echandi, 1965; Godoy-Lutz et al., 1996a; Godoy-Lutz et al., 2003).

The classical hyphal fusion for identification and classification of Rhizoctonia spp. into anastomosis groups has been widely used and is still valid. It has been genetically confirmed in recent years by the use of DNAbased molecular methods. One of the most useful tool is the analysis of sequences from rDNA ITS (Grosch et al. 2004, 2007). This has been used for identification of anastomosis groups. According to Pannecoucque \& Höffe (2009), ITS regions evolve faster and have been used often for molecular identification of closely related fungi. Using this advantage, Godoy-Lutz et al. (2008) developed specific primers for each AG that affected bean. Recently, Wibberg et al. (2013) and Zheng et al. (2013) published the genome of $R$. solani AG 1-IB and AG 1-IA, respectively. All this information can be used to learn about the structure and gene function and to develop phylogeny and diversity studies.

This investigation was conducted to determine the virulence of $R$. solani isolates infecting bean in different climate regions of Costa Rica, and the intraspecific diversity of WB isolates using molecular markers specific for each AG group and ITS sequences.

\section{MATERIALS AND METHODS}

\section{Sampling, isolation and morphological fungus identification}

Leaf samples of bean plants with symptoms of web blight were collected in six climate regions in Costa Rica (South Pacific, Central Valley, North Region, Southern Mountain Region, North Pacific and Caribbean Region) during 2007 and 2009. A global positioning system (GPS) was used to mark the sampling sites. The pathogen was isolated and identified considering the hyphal morphological traits as branching at right angles with constriction at the base and presence of a septum near the point of origin. Monilioid cells and presence of multinucleated cells were checked out and verified (Naito, 1996). Isolates were stored at $4^{\circ} \mathrm{C}$ in Petri dishes on potatodextrose-agar medium (PDA). A photographic database was established, with pictures and a morphological description (mycelium color, size and shape of the sclerotia) of each isolate.

\section{Virulence}

Virulence of the 51 isolates was evaluated on two bean cultivars (Bribrí and Brunca) using the detached leaf method (Steadman et al., 1997). Severity was evaluated 24, 48, and 72 hours post inoculation (h.p.i) using the CIAT 1-9 severity scale, where $1=$ no visible symptoms of disease, $3=$ $5 \%$ of the foliar area infected, $5=10 \%$ of the area infected, $7=25 \%$ of the area infected and $9=>50 \%$ area infected (van Schoonhoven et al., 1987). In each evaluation, photographs were taken to determine the affected area with Scion Image alfa version 4.0.3.2.

\section{Statistical analysis of data}

Analysis of variance was used for isolate comparison, with time as a covariable, and isolate and variety as factors. The interaction between time and isolate was also included. Due to the high interaction between variables, the numerous samples and the low significance of cultivars, isolates were compared without considering cultivar. When differences were encountered, multiple Tukey comparisons were made.

Isolates were classified in three categories according to the virulence level: group A: isolates with average affected leaf areas less than $6.66 \mathrm{~cm}^{2}$; group B: isolates with average areas between $6.66 \mathrm{~cm}^{2}$ and $12.5 \mathrm{~cm}^{2}$; group $\mathrm{C}$ : isolates with averages higher than $12.5 \mathrm{~cm}^{2}$. Each evaluation was compared separately by time and group; however, only the 72 hour evaluations were reported because of their greater importance. Equality of variances and normality of residue, indispensable for the ANOVA, were determined. For the analysis a program was written in $\mathrm{R}$ language version 2.10.0.

\section{DNA extraction and amplification}

DNA was extracted from lyophilized mycelium of $R$. solani, previously cultivated on AC agar (Sigma) with $120 \mu \mathrm{L} / \mathrm{L}(50 \mathrm{mg} / \mathrm{mL})$ of ampicillin, according to the modified protocol by Pascual et al. (2000). Extracted DNA was treated with $5 \mu \mathrm{L}$ RNase A (Fermentas, $10 \mathrm{mg} / \mathrm{mL}$ ) and quantified with a spectrophotometer (Thermo Spectronic Helios Y).

For PCR, a mixture of $25 \mu \mathrm{L}$ was prepared with 0,2 units of Taq DNA polymerase (Dreamtaq, Fermentas), $2 \mu \mathrm{L}$ dNTP mix (25mM), $2,5 \mu \mathrm{L} 10 x$ buffer, $3 \mu \mathrm{L} \mathrm{MgCl}_{2}(25 \mathrm{mM})$, $1,5 \mu \mathrm{L}$ of specific primer for each $\mathrm{AG}(10 \mu \mathrm{m})$ and $50 \mathrm{ng} / \mu \mathrm{L}$ of the DNA sample. The amplification was made using the primers WB-A, WB-B, AG 1-IA, AG 1-IB, AG 1-IC, AG 1-ID, AG 4, AG 2-2, AG 2-2 IIIB and AG 2-2IV (Carling et al. 2002, Godoy-Lutz et al. 2008). Amplifications were performed a PTC-100 thermocycler (MJ Research), with a temperature profile of $95^{\circ} \mathrm{C}$ for 5 min for denaturing, 58$62^{\circ} \mathrm{C}$, for $45 \mathrm{~s}$ for annealing of the primer and $72^{\circ} \mathrm{C}$ for 1 min for extension. A second step consisted of 40 cycles with 
a temperature profile of $95^{\circ} \mathrm{C}$ for $45 \mathrm{~s}$ to denature the DNA, $45 \mathrm{~s}$ at $58-62^{\circ} \mathrm{C}$ for annealing and $1 \mathrm{~min}$ at $72^{\circ} \mathrm{C}$ for extension. The third step consisted of $72^{\circ} \mathrm{C}$ for $10 \mathrm{~min}$. The PCR products were separated by electrophoresis in a $1 \%$ agarose gel in TAE buffer for $90 \mathrm{~min}$ at $100 \mathrm{~V}$. The gel was dyed with ethidium bromide, observed under UV light and photographed with a Kodak EDAS 290 system. A molecular weight marker of 100 bp (50 ng/ $\mu \mathrm{L})$ (Fermentas) was used.

To confirm the relationships among the isolates, 35 isolates were selected at random and amplified using universal primers ITS4 and ITS5. A $25 \mu \mathrm{L}$ PCR mixture was prepared using $0.5 \mu \mathrm{L}$ of Taq DNA polymerase (Dreamtaq, Fermentas), $2.5 \mu \mathrm{L}$ dNTPs mix (25mM), $2.5 \mu \mathrm{L}$ master mix buffer (10x) Fermentas, $0.4 \mu \mathrm{L}$ of each primer $(10 \mu \mathrm{m})$ and $50 \mathrm{ng} / \mu \mathrm{L}$ of the DNA sample. DNA was amplified with the oligonucleotides ITS4 (TCCTCCGCTTATTGATATGC) and ITS5 (GGAAGTAAAAGTCGTAACAAGG) (White et al., 1990) using a PTC-100 thermocycler (MJ Research) with a temperature profile of $94^{\circ} \mathrm{C}$ for $1 \mathrm{~min}$, followed by 30 cycles of $94^{\circ} \mathrm{C}$ for $40 \mathrm{~s}, 55^{\circ} \mathrm{C}$ for $1 \mathrm{~min}$, and $72^{\circ} \mathrm{C}$ for $1 \mathrm{~min}$, and a final step at $72^{\circ} \mathrm{C}$ for $5 \mathrm{~min}$. PCR products of $740 \mathrm{bp}$ were visualized as indicated before. PCR products were purified with the QIAquick PCR Purification Kit (Qiagen) and sequenced using both primer strands by using the BigDyeTerminator v.3.1 cycle sequencing kit (Applied Biosystems) and an ABI Prism 3130 (Applied Biosystems). With the identification of each anastomosis subgroup and the GPS data, a map was constructed using Arcview GIS 3.2.

Sequence data for the complete ITS-5.8S rDNA of isolates of AG 1 and AG 2 and AG 4 was manually checked and edited using the Multiple Sequence Alignment of BioEdit software (Hall, 2007). Sequences of the complete ITS-5.8S rDNA region were compared with nine reference sequences (NCBI GenBank): AJ868444 (AG 1), AF308624 (AG 2-2), AF308623 (AG 2 WB), JF519829 (AG P), AB195928 (AG 1-IA), AB122139 (AG 1-IB), AB122141 (AG 1-IC), EF197798 (AG 1-ID), JF946728 (AG 1-IE) and JF946731 (AG 1-IF), JQ669932 (AG4).

All nucleotide substitutions were equally weighted and unordered. Alignment gaps were treated as missing data. The sequences were analyzed with BLAST for confirmation of the AG, aligned with the MAFFT algorithm in the GUIDANCE server and analyzed with MrBayes3.2 using 1,000,000 Markov chains (Ronquist et al., 2011) for phylogenetic tree construction with a GTR model. The tree was visualized in MEGA5.2 (Tamura et al., 2011). To ensure the robustness of the analysis additional trees were constructed using Neighbor joining (NJ) and Maximum likelihood (ML) methods with 5,000 bootstrap replications using MEGA 5.2.

\section{RESULTS}

\section{Sampling, isolation and identification}

During 2007-2009, foliar bean tissue infected with $R$. solani was collected throughout the national territory. Anastomosis subgroup, sclerotium size, GenBank accession, host, altitude, climate subregions and collecting year for each isolate are presented in Table 1. Fifty-one isolates of Rhizoctonia solani were obtained. The mycelium coloration varied in intensity from a dark brown to beige and/or white. Sclerotia size varied from 5 to $20 \mathrm{~mm}$, for macrosclerotia single or aggregated; and $1 \mathrm{~mm}$ for isolates with microsclerotia. Also all those mycelium presented multinucleated cells and near-right-angle branching pattern.

\section{Virulence}

The CIAT scale scores were compared with measured affected areas for each isolate and a linear correlation of 0.46 was obtained. Although significant, the value was lower than expected. Since the CIAT scale is subjective, these data were not used and conclusions were based on average measured affected leaf areas.

Group A was characterized by a number of heterogeneous isolates $(p<0.0001,27,350)$ (Figure 1). Tukey comparisons showed differences among isolates when analyzed by pairs. In group B, significant differences in average areas were observed between isolates $(\mathrm{p}<0.0001$, $16,264)$ however a great homogeneity was observed. This group included 17 isolates and the Tukey comparisons showed differences between isolate 3 and those with lower averages.

Group C was homogenous at every evaluation time, as observed in the Figure 2. The ANOVA confirmed that there was no significant difference between average areas of different isolates at the 72 hour evaluations $(p=0.13,7$, 119). Isolates $3843,3636,3233,3801,3242,3841$ and 3804 are the members of this group. These seven isolates are AG 1 , but belong to different subgroups and climate regions. Only isolate 3979 belongs to AG 2-2WB.

\section{Molecular identification of the AGs}

A high degree of variability between isolates was determined using specific primers for each $A G$ and subgroup. AG groups AG 1, AG 2 and AG 4 were identified in different climate regions of Costa Rica using specific primers for each AG. Subgroups AG 1-IA, AG 1-IB, AG 1-ID, AG 1-IE, AG 1-IF, AG 2WB and AG 4 were identified. The AG was independent of geographical origin and climate region (Table 1).

A map with the distribution of the anastomosis subgroup was constructed. In the Figure 3 there is possible to observe that AG 1-IE subgroup was found with the greatest frequency in the different climate subregions, which are characterized by differences in altitude and precipitation. A single isolate of AG 1-1A was identified in a sample from the South Pacific region. AG 1-1B was found in the South Pacific, Central Valley and Northern regions.

ITS sequences confirm previous results obtained with specific primers. Also, Costa Rican isolates 3977, 3625 and 3629 corresponded to AG P, which has not 
TABLE 1 - Code, anastomosis subgroup (AG), sclerotium size, GenBank accession, host, altitude (meters above sea level), climate subregions and collecting year of 51 isolates of Rhizoctonia solani collected in different geographic regions of Costa Rica and reference accessions used for phylogenetic tree construction obtained from GenBank

\begin{tabular}{|c|c|c|c|c|c|c|c|}
\hline Code & $\begin{array}{l}\text { Anastomosis } \\
\text { subgroup }\end{array}$ & Sclerotium size & $\begin{array}{l}\text { GenBank } \\
\text { accession } \\
\text { number }\end{array}$ & Host & Altitude & $\begin{array}{l}\text { Climate } \\
\text { subregions }\end{array}$ & Year \\
\hline 3979 & AG 1-IE & Macrosclerotia & JX294346 & $\begin{array}{l}\text { P. vulgaris cv. } \\
\text { Mantequilla }\end{array}$ & 949 & North Pacific & 2008 \\
\hline 3977 & AG P & Microsclerotia & JX294345 & $\begin{array}{l}\text { P. vulgaris } \mathrm{cv} \text {. } \\
\text { unknown }\end{array}$ & 1000 & North Pacific & 2008 \\
\hline G14 & AG 4 & Microsclerotia & JX294349 & $\begin{array}{l}\text { P. vulgaris } \mathrm{cv} \text {. } \\
\text { unknown }\end{array}$ & 1233 & North Pacific & 2009 \\
\hline 4161 & AG 2 WB & Microsclerotia & JX294348 & $\begin{array}{l}\text { P. vulgaris cv. Don } \\
\text { Pedro }\end{array}$ & 1249 & North Pacific & 2008 \\
\hline 4160 & AG 2 WB & Macrosclerotia & JX294347 & $\begin{array}{l}\text { P. vulgaris } \mathrm{cv} \text {. } \\
\text { Chimbolo }\end{array}$ & 1203 & North Pacific & 2009 \\
\hline $9 \mathrm{D}$ & AG $2 \mathrm{WB}$ & Macrosclerotia & & $\begin{array}{l}\text { P. vulgaris cv. } \\
\text { unknown }\end{array}$ & 1229 & North Pacific & 2009 \\
\hline $11 \mathrm{D}$ & AG $2 \mathrm{WB}$ & Macrosclerotia & JX294316 & $\begin{array}{l}\text { P. vulgaris cv. } \\
\text { unknown }\end{array}$ & 1203 & North Pacific & 2009 \\
\hline 3812 & AG 1-IA & Microsclerotia & JX294340 & $\begin{array}{l}\text { P. vulgaris cv. } \\
\text { unknown }\end{array}$ & 1647 & South Pacific & 2008 \\
\hline 3307 & AG $2 W B$ & Macrosclerotia & & $\begin{array}{l}\text { P. vulgaris } \mathrm{cv} \text {. } \\
\text { unknown }\end{array}$ & 1600 & South Pacific & 2007 \\
\hline 3796 & AG 1-IE & Macrosclerotia & JX294331 & $\begin{array}{l}\text { P. vulgaris cv. } \\
\text { unknown }\end{array}$ & 1600 & South Pacific & 2008 \\
\hline 3791 & AG 1-IE & Macrosclerotia & JX294328 & $\begin{array}{l}\text { P. vulgaris } \mathrm{cv} \text {. } \\
\text { unknown }\end{array}$ & 1600 & South Pacific & 2008 \\
\hline 3800 & AG 1-IE & Macrosclerotia & JX294333 & $\begin{array}{l}\text { P. vulgaris } \mathrm{cv} \text {. } \\
\text { unknown }\end{array}$ & 1645 & South Pacific & 2008 \\
\hline 3810 & AG 1-IA & Macrosclerotia & JX294339 & $\begin{array}{l}\text { P. vulgaris cv. } \\
\text { unknown }\end{array}$ & 1642 & South Pacific & 2008 \\
\hline 3798 & AG 1-IF & Microsclerotia & JX294332 & $\begin{array}{l}\text { P. vulgaris } \mathrm{cv} \text {. } \\
\text { unknown }\end{array}$ & 1600 & South Pacific & 2008 \\
\hline 3794 & AG 1-IE & Macrosclerotia & JX294329 & $\begin{array}{l}\text { P. vulgaris } \mathrm{cv} \text {. } \\
\text { Kiura Colorado }\end{array}$ & 1600 & South Pacific & 2008 \\
\hline 3240 & AG 1-IE & Macrosclerotia & JX294318 & $\begin{array}{l}\text { P. vulgaris cv. } \\
\text { unknown }\end{array}$ & 1600 & South Pacific & 2007 \\
\hline 3233 & AG 1-IE & Macrosclerotia & & $\begin{array}{l}\text { P. vulgaris } \mathrm{cv} \text {. } \\
\text { unknown }\end{array}$ & 1600 & South Pacific & 2007 \\
\hline 3801 & AG 1-IE & Macrosclerotia & JX294334 & $\begin{array}{l}\text { P. vulgaris var. } \\
\text { Brunca }\end{array}$ & 1645 & South Pacific & 2008 \\
\hline 3803 & AG 1-IF & Microsclerotia & JX294335 & $\begin{array}{l}\text { P. vulgaris cv. } \\
\text { Green bean }\end{array}$ & 1640 & South Pacific & 2008 \\
\hline 3792 & AG 1-IF & Microsclerotia & & $\begin{array}{l}P . \text { vulgaris } \mathrm{cv} \text {. } \\
\text { unknown }\end{array}$ & 1600 & South Pacific & 2008 \\
\hline 3811 & AG 2 WB & Microsclerotia & & $\begin{array}{l}\text { P. vulgaris } \mathrm{cv} . \\
\text { Poroto amarillo }\end{array}$ & 1640 & South Pacific & 2008 \\
\hline 3838 & AG 2 WB & Microsclerotia & JX294341 & $\begin{array}{l}\text { P. vulgaris cv. } \\
\text { unknown }\end{array}$ & 1600 & South Pacific & 2007 \\
\hline 3708 & AG $2 \mathrm{WB}$ & Microsclerotia & & $\begin{array}{l}\text { P. vulgaris cv. } \\
\text { unknown }\end{array}$ & 1642 & South Pacific & 2008 \\
\hline 3249 & AG4 & Microsclerotia & JX294320 & $\begin{array}{l}\text { P. vulgaris cv. } \\
\text { unknown }\end{array}$ & 1600 & South Pacific & 2007 \\
\hline 3845 & AG 1-ID & Microsclerotia & JX294344 & $\begin{array}{l}\text { P. vulgaris cv. } \\
\text { Cuba }\end{array}$ & 1138 & $\begin{array}{l}\text { Caribbean } \\
\text { Region }\end{array}$ & 2008 \\
\hline 3236 & AG 1-IE & Macrosclerotia & & $\begin{array}{l}\text { P. vulgaris var. } \\
\text { Cabecar }\end{array}$ & 600 & $\begin{array}{l}\text { Caribbean } \\
\text { Region }\end{array}$ & 2007 \\
\hline 3847 & AG 2 WB & Macrosclerotia & & $\begin{array}{l}\text { P. vulgaris } \mathrm{cv} \text {. } \\
\text { unknown }\end{array}$ & 1149 & $\begin{array}{l}\text { Caribbean } \\
\text { Region }\end{array}$ & 2007 \\
\hline 3628 & AG 1-IE & Macrosclerotia & JX294324 & $\begin{array}{l}\text { P. vulgaris cv. } \\
\text { unknown }\end{array}$ & 1000 & $\begin{array}{l}\text { Southern } \\
\text { Mountain } \\
\text { Region }\end{array}$ & 2008 \\
\hline
\end{tabular}


Cont.

\begin{tabular}{|c|c|c|c|c|c|c|c|}
\hline Code & $\begin{array}{l}\text { Anastomosis } \\
\text { subgroup }\end{array}$ & Sclerotium size & $\begin{array}{l}\text { GenBank } \\
\text { accession } \\
\text { number }\end{array}$ & Host & Altitude & $\begin{array}{l}\text { Climate } \\
\text { subregions }\end{array}$ & Year \\
\hline 3628 & AG 1-IE & Macrosclerotia & JX294324 & $\begin{array}{l}\text { P. vulgaris cv. } \\
\text { unknown }\end{array}$ & 1000 & $\begin{array}{l}\text { Southern } \\
\text { Mountain } \\
\text { Region }\end{array}$ & 2008 \\
\hline 3626 & AG 1-IE & Macrosclerotia & JX294323 & $\begin{array}{l}\text { P. vulgaris } \mathrm{cv} \text {. } \\
\text { Landrace }\end{array}$ & 1125 & $\begin{array}{l}\text { Southern } \\
\text { Mountain } \\
\text { Region }\end{array}$ & 2008 \\
\hline 3636 & AG 1-IE & Macrosclerotia & & $\begin{array}{l}\text { P. vulgaris cv. } \\
\text { unknown }\end{array}$ & 1000 & $\begin{array}{l}\text { Southern } \\
\text { Mountain } \\
\text { Region }\end{array}$ & 2008 \\
\hline 3242 & AG 1-IF & Microsclerotia & JX294319 & $\begin{array}{l}\text { P. vulgaris cv. } \\
\text { unknown }\end{array}$ & 1600 & $\begin{array}{l}\text { Southern } \\
\text { Mountain } \\
\text { Region }\end{array}$ & 2007 \\
\hline 3625 & AG P & Macrosclerotia & JX294322 & $\begin{array}{l}\text { P. vulgaris cv. } \\
\text { Mantequilla }\end{array}$ & 1000 & $\begin{array}{l}\text { Southern } \\
\text { Mountain } \\
\text { Region }\end{array}$ & 2008 \\
\hline 3629 & AG P & Microsclerotia & JX294325 & $\begin{array}{l}\text { P. vulgaris var. } \\
\text { Mexico } 80\end{array}$ & 1125 & $\begin{array}{l}\text { Southern } \\
\text { Mountain } \\
\text { Region }\end{array}$ & 2008 \\
\hline 3668 & AG 1-IB & Macrosclerotia & & $\begin{array}{l}\text { P. vulgaris cv. } \\
\text { unknown }\end{array}$ & 543 & $\begin{array}{l}\text { Southern } \\
\text { Mountain } \\
\text { Region }\end{array}$ & 2008 \\
\hline 3620 & AG 2 WB & Microsclerotia & JX294321 & $\begin{array}{l}\text { P. vulgaris cv. } \\
\text { unknown }\end{array}$ & 1097 & $\begin{array}{l}\text { Southern } \\
\text { Mountain } \\
\text { Region }\end{array}$ & 2008 \\
\hline 3806 & AG 1-IA & Macrosclerotia & JX294336 & $\begin{array}{l}\text { P. vulgaris } \mathrm{cv} \text {. } \\
\text { unknown }\end{array}$ & 1107 & $\begin{array}{l}\text { Northern } \\
\text { Region }\end{array}$ & 2008 \\
\hline 3804 & AG 1-IB & Microsclerotia & & $\begin{array}{l}\text { P. vulgaris cv. } \\
\text { unknown }\end{array}$ & 1214 & $\begin{array}{l}\text { Northern } \\
\text { Region }\end{array}$ & 2008 \\
\hline 3808 & AG 1-IE & Macrosclerotia & JX294338 & $\begin{array}{l}\text { P. vulgaris cv. } \\
\text { unknown }\end{array}$ & 1202 & $\begin{array}{l}\text { Northern } \\
\text { Region }\end{array}$ & 2008 \\
\hline 3077 & AG 1-IE & Macrosclerotia & & $\begin{array}{l}\text { P. vulgaris } \mathrm{cv} \text {. } \\
\text { unknown }\end{array}$ & 1000 & $\begin{array}{l}\text { Northern } \\
\text { Region }\end{array}$ & 2007 \\
\hline 3093 & AG 1-IE & Macrosclerotia & JX294317 & $\begin{array}{l}\text { P. vulgaris cv. } \\
\text { unknown }\end{array}$ & 1000 & $\begin{array}{l}\text { Northern } \\
\text { Region }\end{array}$ & 2007 \\
\hline 3840 & AG 1-IF & Microsclerotia & & $\begin{array}{l}\text { P. vu lgaris cv. } \\
\text { unknown }\end{array}$ & 1236 & $\begin{array}{l}\text { Northern } \\
\text { Region }\end{array}$ & 2008 \\
\hline 3841 & AG 1-IB & Microsclerotia & JX294342 & $\begin{array}{l}\text { P. vulgaris } \mathrm{cv} \text {. } \\
\text { unknown }\end{array}$ & 1123 & Central Valley & 2008 \\
\hline 3843 & AG 1-IE & Macrosclerotia & JX294343 & $\begin{array}{l}\text { P. vulgaris cv. } \\
\text { unknown }\end{array}$ & 600 & Central Valley & 2008 \\
\hline 3973 & AG 1-IE & Macrosclerotia & & $\begin{array}{l}\text { P. vulgaris cv. } \\
\text { unknown }\end{array}$ & 1042 & Central Valley & 2008 \\
\hline Cach & AG 2 WB & Microsclerotia & & $\begin{array}{l}\text { P. vulgaris cv. } \\
\text { unknown }\end{array}$ & 1042 & Central Valley & 2008 \\
\hline 3677 & AG 1-IE & Macrosclerotia & JX294326 & $\begin{array}{l}\text { P. vulgaris cv. } \\
\text { unknown }\end{array}$ & 543 & Central Valley & 2008 \\
\hline 3667 & AG 1-IE & Macrosclerotia & & $\begin{array}{l}\text { P. vulgaris cv. } \\
\text { Landrace }\end{array}$ & 700 & Central Valley & 2008 \\
\hline 3793 & AG 1-IF & Microsclerotia & & $\begin{array}{l}\text { P. vulgaris cv. } \\
\text { unknown }\end{array}$ & 700 & Central Valley & 2008 \\
\hline 3795 & AG 1-IF & Microsclerotia & JX294330 & $\begin{array}{l}\text { P. vulgaris cv. } \\
\text { unknown }\end{array}$ & 1470 & Central Valley & 2008 \\
\hline
\end{tabular}


Cont.

\begin{tabular}{|c|c|c|c|c|c|c|c|}
\hline Code & $\begin{array}{l}\text { Anastomosis } \\
\text { subgroup }\end{array}$ & Sclerotium size & $\begin{array}{l}\text { GenBank } \\
\text { accession } \\
\text { number }\end{array}$ & Host & Altitude & $\begin{array}{l}\text { Climate } \\
\text { subregions }\end{array}$ & Year \\
\hline 3807 & AG 2 WB & Macrosclerotia & JX294337 & $\begin{array}{l}\text { P. vulgaris } \mathrm{cv} \text {. } \\
\text { unknown }\end{array}$ & 646 & Central Valley & 2008 \\
\hline \multirow[t]{12}{*}{3809} & AG 2 WB & Macrosclerotia & & $\begin{array}{l}\text { P. vulgaris cv. } \\
\text { unknown }\end{array}$ & 646 & Central Valley & 2008 \\
\hline & AG 1-IB & & AJ868444 & & & & \\
\hline & AG 2-2 & & AF308624 & & & & \\
\hline & AG 2 WB & & AF308623 & & & & \\
\hline & AG P & & JF519829 & & & & \\
\hline & AG 1-IA & & AB195928 & & & & \\
\hline & AG 1-IB & & AB122139 & & & & \\
\hline & AG 1-IC & & AB122141 & & & & \\
\hline & AG 1-ID & & EF197798 & & & & \\
\hline & AG 1-IE & & JF946728 & & & & \\
\hline & AG 1-IF & & JF946731 & & & & \\
\hline & $\mathrm{AG} 4$ & & JQ669932 & & & & \\
\hline
\end{tabular}

Group A

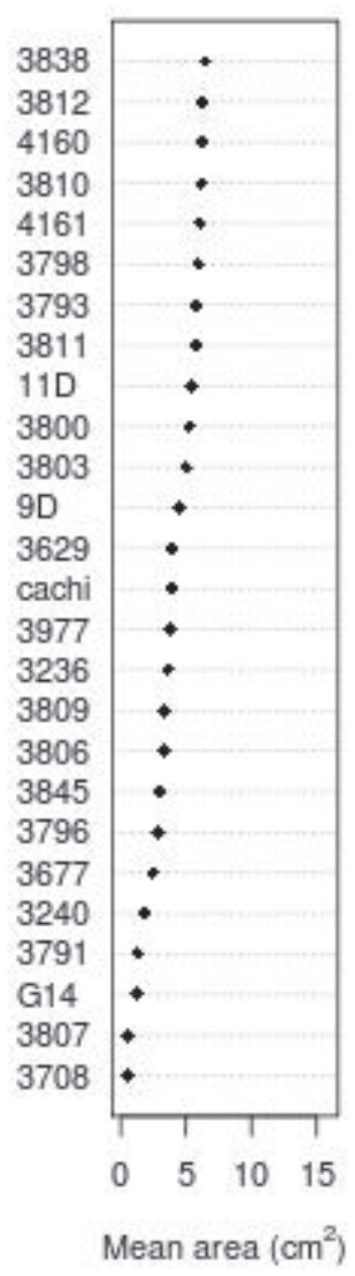

Group B

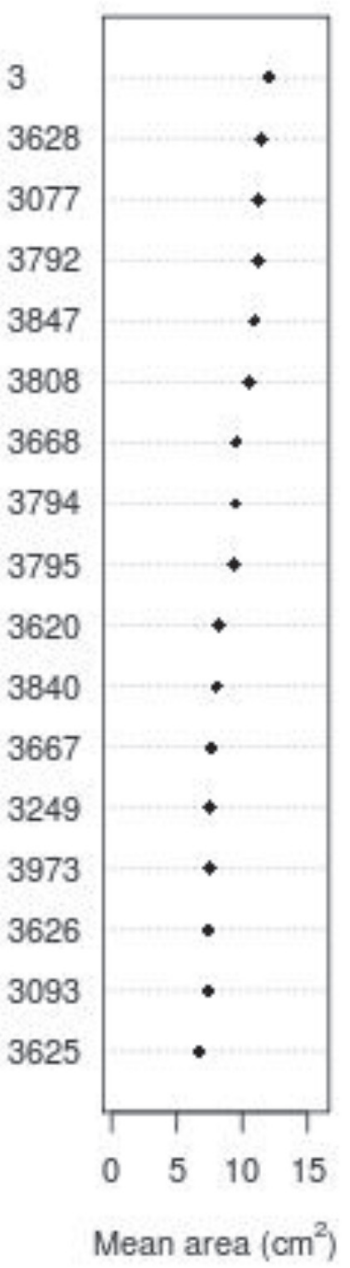

Group C

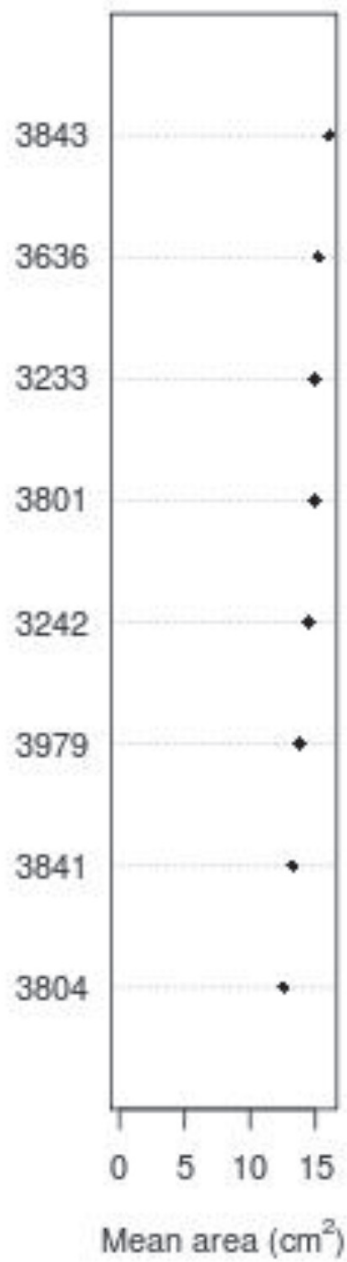

FIGURE 1 - Degree of virulence (average affected area) of Rhizoctonia solani isolates inoculated in vivo on detached bean leaves, evaluated at 72 hours post inoculation (h.p.i) according to virulence groups: A, low; B, medium; C, high. 
Virulence and molecular characterization of Costa Rican isolates of Rhizoctonia solani from common bean

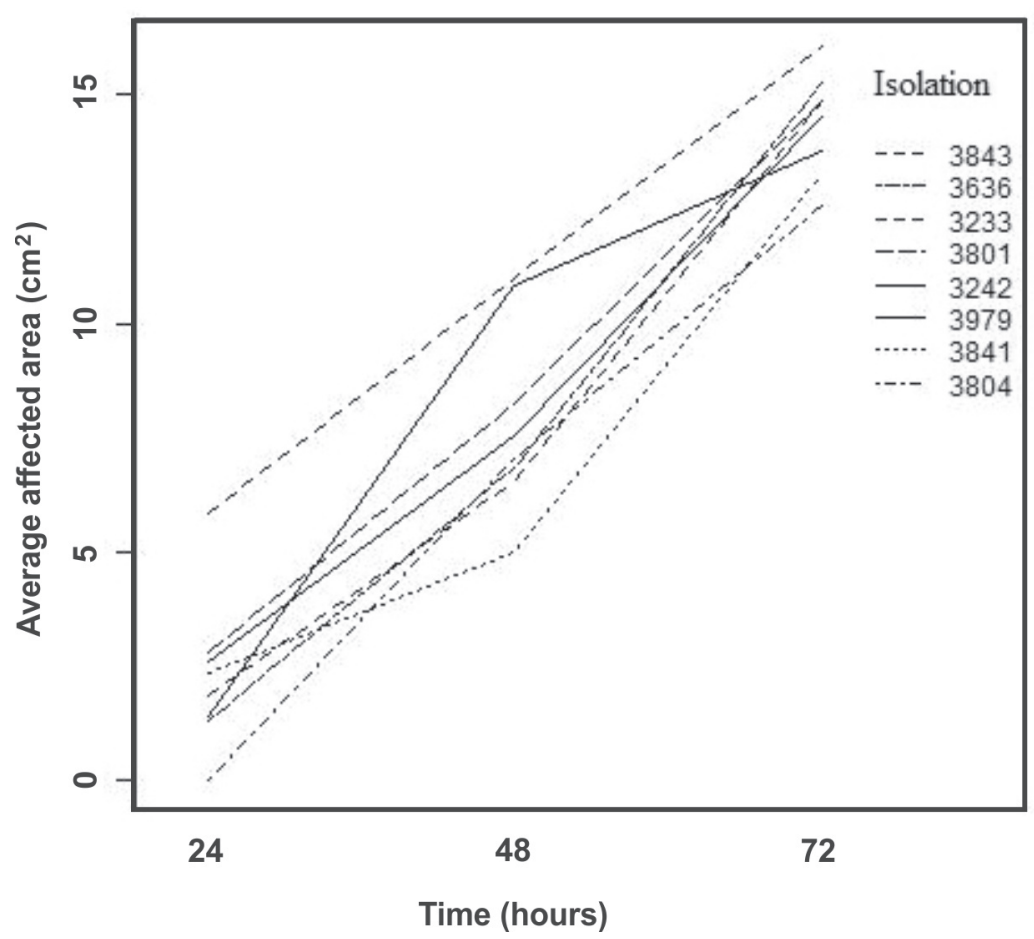

FIGURE 2 - Average affected area $\left(\mathrm{cm}^{2}\right)$ by the most virulent isolates (group C) at 24,48 and 72 hours.

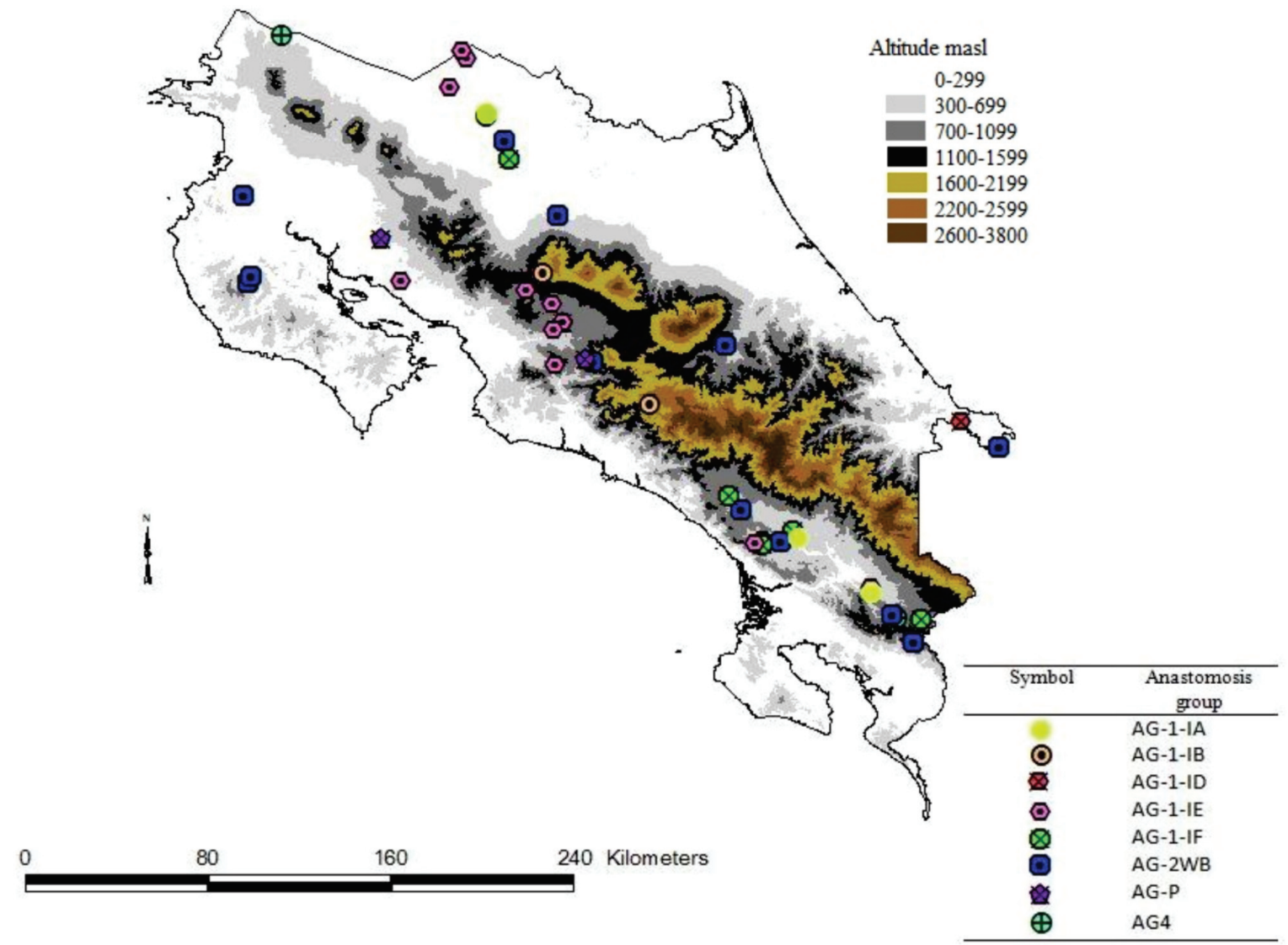

FIGURE 3 - Costa Rica map and anastomosis group distribution by altitude (meters above sea level). 


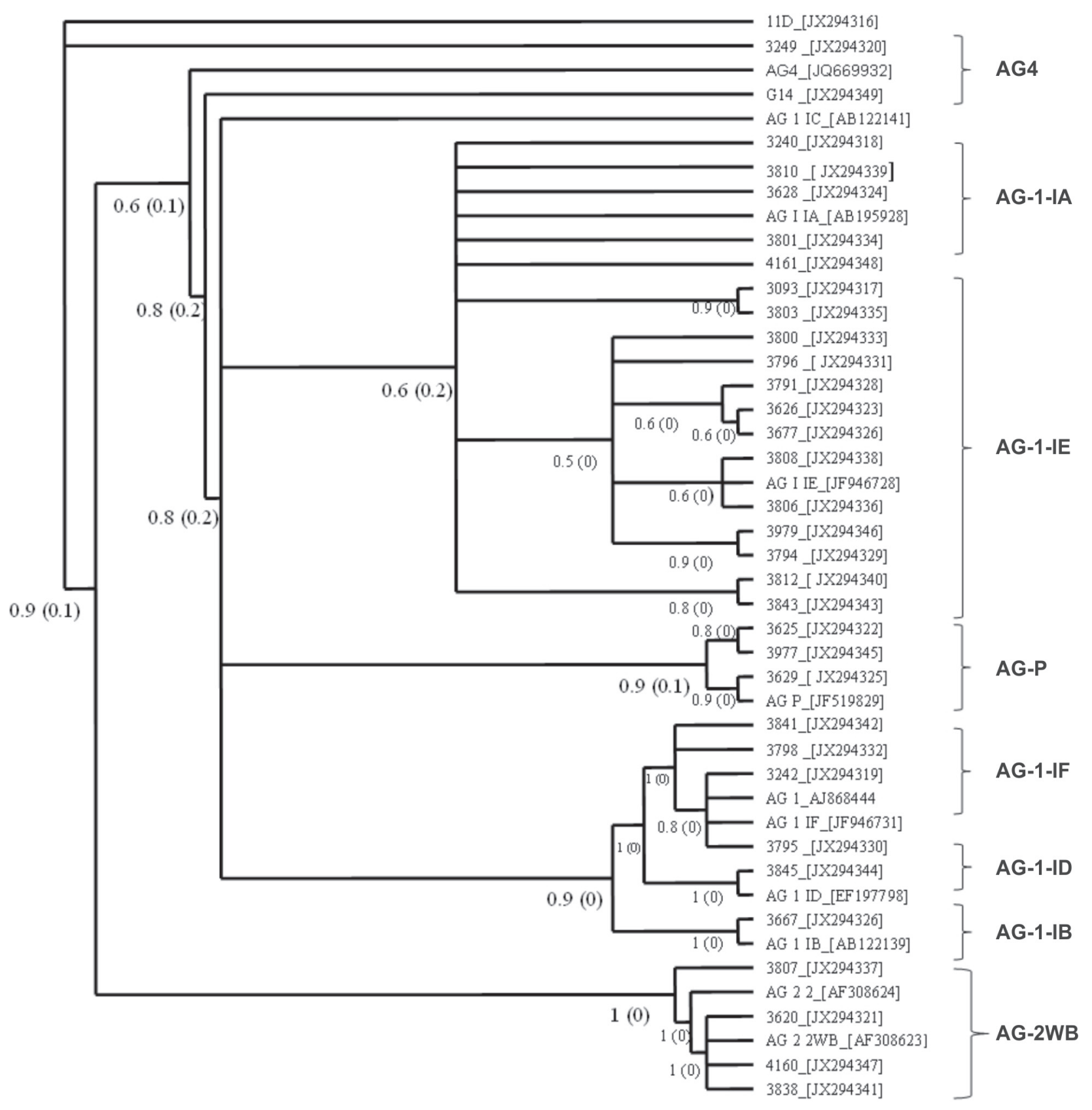

FIGURE 4 - Phylogenetic analysis using Bayesian inference based on ITS sequences, using MrBayes 3.2 with 1,000,000 Markov chains, with a multiple sequence alignment with the MAFFT algorithm on the GUIDANCE server.

previously been reported in P. vulgaris. The phylogenetic tree (Figure 4) confirmed the grouping of isolates in two clades. The first clade contained accessions of AG 4 and AG $2 \mathrm{WB}$ from diverse climate regions. Bootstrap values from $60-100 \%$ allowed subgroup separation. Accessions of the AG 1 subgroup were included in second clade. This was a heterogeneous group that included AG 1-ID, AG 1-IE, AG 1-IF and AG P from different geographical regions of the country. NJ and MP trees were constructed (data not shown) and similar results were found.

\section{DISCUSSION}

Fifty-one isolates of $R$. solani were obtained from symptomatic leaves of common bean from different climate regions of Costa Rica. Isolates were characterized by size of sclerotia (macro or micro). Godoy-Lutz et al. (2003) point out the importance of mycelium and sclerotia in the epidemiology of the disease, as they constitute the main source of primary inoculum. AG 1-IF and AG 1-IE differ in sclerotia size and culture pigmentation. These 
characteristics could be a key for distinguishing between isolates (Godoy-Lutz et al., 2003). The different phenotypes found in this study is indicative of the high variation that this fungi presents in our study.

Of the 51 isolates, 18 were from the South Pacific Region, and seven from the Northern Pacific Region (Table 1), both areas of major bean production in Costa Rica. The lowest number of isolates was found in the Caribbean, which is not a bean producing region and it is located near the Atlantic shore. The isolates with the highest virulence according to the CIAT scale and the measured area were placed in group C. Isolate 3843 was considered the most virulent at 24 h.p.i, because it caused the highest amount of affected foliar tissue in the shortest time period. At 48 hours there was no more healthy foliar tissue to infect, so at 72 hours, the affected area was similar to the other isolates. This isolate is characterized by macroesclerotia. Godoy-Lutz et al. (2003) mention that AG 1 macrosclerotia producing isolates were the most virulent, and AG 4 no if you read well is AG 2-2 the least virulent. Similar results have been observed in Latin America and the Caribbean, where virulence patterns vary between regions and genotypes (González-Vera et al., 2010).

Diverse levels of virulence between and within the identified groups were observed. The most virulent isolate (3843) was identified as AG 1-IE, and the least aggressive isolate was AG 2 WB. Isolate 3843 was from the Central Valley region, an area with precipitation from 1500 to $2500 \mathrm{~mm}^{3}$ and average temperatures between 19 and $22^{\circ} \mathrm{C}$. With these data one might infer that these are favorable conditions for the disease development. Beaver et al. (2002) report differences in virulence on common bean using isolates of $R$. solani from Central America and the Caribbean. Lee et al. (2006) mention that the virulence of $R$. solani can be affected by environmental conditions present at the beginning of the infection.

No relation was observed between virulence, $A G$ and geographical origin. Different AGs and subgroups were present in all of the regions sampled, regardless of edaphic and climatic conditions. This demonstrates the great adaptability and survival capacity of this pathogen, an important consideration in breeding $P$. vulgaris for resistance. The variable genetic composition of the fungus can affect the dynamics of the pathogen-host relationship, as pathogens are able to adapt to specific ecological niches in diverse agrosystems (Araya, 2003).

Results of this study show that WB in common bean in Costa Rica is caused at least by four AGs, AG 1, AG 2, AG 4 and AG P, and diverse subgroups, AG 1-IA, AG 1-IB, AG 1-IC, AG 1-ID, AG 1-IE, AG 1-IF, AG 2-2WB and $A G 4$. The dominant groups in this study were $A G 1$ $(65 \%)$ and the AG 1-IE subgroup (37\%). AG 1-IE and AG 2 WB subgroups were found in every climate region sampled. AG 1-IE was the most frequent (Table 1). These results are consistent with reports in the literature that show a prevalence of this subgroup in field populations causing WB in common bean in several Latin American and Caribbean countries (Echandi, 1965; Godoy-Lutz et al., 1996b).

The presence of different AGs found in this study in different geographical regions suggests that bean varieties are susceptible to a broad range of AG subgroups (GodoyLutz et al., 2008). The presence of AG P, not previously reported in bean, in the North Pacific and Southern Mountain regions, suggests that new AGs may be appearing. AG P is a heterogeneous group, as shown by the percent similarity between isolates of this AG using MP and NJ methods. For some isolates, similarity ranged from $93 \%$ to $100 \%$, but for others it was lower, from $90 \%$ to $94 \%$. This AG may include several subgroups, but additional work with more isolates is needed to confirm the existence of AG P subgroups (Sharon et al., 2008).

Identification with specific primers aids in determining the prevalence of an $A G$ or subgroup in a certain geographic area. Population genetics of this pathogenic fungus may be affected by diverse mechanisms not yet elucidated. Since in this study AGs were not related to place of origin, it appears that many mechanisms, such as mating systems, reproduction strategies and gene flow, could be contributing to pathogen dispersal (González et al., 2006). Bean programs in Costa Rica in collaboration with farmers associations have been done during the last years to provide certified seeds. However, the presence of anastomosis subgroups between regions could be an indicative that contaminated seed movement still spreads in the country. Godoy-Lutz et al. (2003) affirm that gene flow may be occurring through seed movement as the pathogen can be spread on asymptomatic seeds (Godoy-Lutz, 2003).

One of the most important results of this research was to confirm the presence of different anastomosis subgroups in the country and their virulence. Systematical sampling of open field crops is necessary to generate recommendations for disease management such as use of crop rotations. According to Okubara et al. (2008) there are voids in the knowledge of pathogen dispersal, crop loss and the role of environmental conditions such as rain and temperature on pathogen populations.

The variable genetic composition of $R$. solani can be affected by the dynamics of the host-pathogen relationship, geneticflexibility and the degree of adaptation to the ecological niche in diverse agroecosystems (Godoy-Lutz et al., 2008). Our phylogenetic tree shows that some anastomosis subgroups are not closely related to the controls. This may be an indication of the mentioned plasticity of the genetic composition of the $R$. solani AG subgroups since they present higher sequence variability than other isolates of $R$. solani. AG P is closer to AG 1-IC. AG P clusters may split into several clusters. Some AG P and AG S, R and Fa clusters have appeared between $A G$ 2-1, AG 2-2 and AG 12. Other AG P isolates cluster together with $\mathrm{AGs} F b$ and $\mathrm{E}$ and are between $\mathrm{AG}$ 6, AG 1-IC and AG 7 subgroups in some cases (Sharon et al., 2008). 
The support percentages of the Bayesian analysis ranged from $60 \%$ to $100 \%$, most of the results were supported by a high value. This supports the tree structure for these isolates and their identification within an $A G$ group or subgroup. Previous studies have shown that AG 4 and AG 2-WB have a close phylogenetical relationship, supporting our hypothesis presented on the tree. In this case, the sequences used were 5.8S rDNA with flanking sequences of ITS1 and ITS2. Sharon et al. (2008) affirm that there is a difference between ITS 1 and ITS2 in terms of variability. ITS 1 sequences are more variable than ITS2 among fungal isolates, but the combined 3 sequences ITS1 $+5.8 \mathrm{~S}+$ ITS 2 used in this study give more information than either sequence separately.

Proper deployment of molecular methods allows the identification and over time monitoring of subgroups associated with the disease in high epidemic risk areas and provides an important tool for analysis of isolate variation in the field and the possible generation of more pathogenic AG subgroups. Knowledge of virulence variation and distribution of the AGs in the country is useful in the assessment of management and breeding strategies in Costa Rica.

\section{ACKNOWLEDGEMENTS}

The authors would like to acknowledge the Consejo Nacional de Rectores (CONICIT), Vicerrectoría de Investigacion, Universidad de Costa Rica and University of Nebraska-Lincoln.

\section{REFERENCES}

Araya CM (2003) Coevolución de interacciones hospedantepatógeno en frijol común. Fitopatologia Brasileira 28:221-228.

Beaver J, Godoy G, Rosas JC, Steadman JR (2002) Estrategia para la seleccionar frijol común con mayor resistencia a mustia hilachosa. Agronomía Mesoamericana 13:67-72.

Beebe SE, Pastor-Corrales MA (1991) Breeding for disease resistance. In: van Schoonhoven A, Voyest O (Eds.) Common beans: Research for crop improvement. Wallingford UK. CAB International. pp. 561-617.

Carling DE (1996) First report of powdery scab of potatoes in Alaska. Plant Disease 80:1208.

Carling DE, Pope EJ, Brainard KA, Carter DA (1999) Characterization of mycorrhizal isolates of Rhizoctonia solani from an orchid, including AG 12, a new anastomosis group. Phytopathology 89:942-946.

Carling DE, Kuninaga S, Brainard KA (2002) Hyphal anastomosis reactions, rDNA-internal transcribed spacer sequences, and virulence levels among subsets of Rhizoctonia solani Anastomosis Group 2 (AG 2) and AG BI. Phytopathology 92:43-50.

Echandi E (1965) Basidiospore infection by Pellicularia filamentosa (=Corticium microsclerotia), the incitant of web blight of common bean. Phytopathology 55:698-99.
Food and Agriculture Organization - FAO (2004) Production Yearbook 2004. Rome Italy. FAO.

Godoy-Lutz G, Arias J, Steadman JR, Eskridge KM (1996a) The web blight pathogen: Its effect on common bean seed quality, germination and early disease development. Annual Report of the Bean Improvement Cooperative 39:152-153.

Godoy-Lutz G, Arias J, Saladin F, Steadman JR, Carling DE (1996b) Characterization of isolates of $R$. solani that cause web blight of common beans in Central America and the Caribbean with implications for disease management. Annual Report of the Bean Improvement Cooperative 39:154-155.

Godoy-Lutz G, Steadman J, Powers R, Higgins B (2000) DNA variation and virulence among isolates causing web blight on common beans. Annual Report of the Bean Improvement Cooperative 43:72-73.

Godoy-Lutz G, Steadman J, Higgins B, Powers, K (2003) Genetic variation among isolates of the web blight pathogen of common bean based on PCR-RFLP of the ITS-rDNA Region. Plant Disease 87:766-771.

Godoy-Lutz G, Kuninaga S, Steadman R, Powers K (2008) Phylogenetic analysis of Rhizoctonia solani subgroups associated with web blight symptoms on common bean based on ITS-5.8S rDNA. Journal of General Plant Pathology 74:32-40.

González D, Cubeta MA, Vilgalys R (2006) Phylogenetic utility of indels within ribosomal DNA and $\beta$-tubulin sequences from fungi in the Rhizoctonia solani species complex. Molecular Phylogenetics and Evolution 40:459-470.

González-Vera AD, Bernardes-de-Assis J, Zala M, McDonald BA, Correa-Victoria F, Graterol-Matute EJ, Ceresini PC (2010) Divergence between sympatric rice- and maize-infecting populations of Rhizoctonia solani AG 1-IA from Latin America. Phytopathology 100:172-182.

González N, Godoy-Lutz G, Steadman JR, Higgins R, Eskridge KM (2012) Assessing genetic diversity in the web blight pathogen Thanatephorus cucumeris (anamorph = Rhizoctonia solani) subgroups AG 1-IE and AG 1-IF with molecular markers. Journal of General Plant Pathology 78:85-98.

Grosch R, Schneider JHM, Kofoet A (2004) Characterisation of Rhizoctonia solani anastomosis groups causing bottom rot in field grown lettuce in Germany. European Journal of Plant Pathology 110:53-62.

Grosch R, Schneider JHM, Peth A, Waschke A, Franken P, Kofoet A, Jabaji-Hare SH (2007) Development of a specific PCR assay for the detection of Rhizoctonia solani AG 1-IB using SCAR primers. Journal of Applied Microbiology 102:806-819.

Hall T (2007) BioEdit v7.0.9. Available at: http://www.mbio.ncsu. edu/BioEdit/ page2.html. Accessed on October 12, 2012.

Kanini GS, Katsifas EA, Savvides AL, Hatzinikolaou DG, Karagouni AD (2013) Greek indigenous streptomycetes as biocontrol agents against the soil-borne fungal plant pathogen Rhizoctonia solani. Journal of Applied Microbiology 114:14681479.

Lee J, Bricker TM, Lefevre M, Pinson S, Oard J (2006) Proteomic and genetic approaches to identifying defence-related proteins in rice challenged with the fungal pathogen Rhizoctonia solani. Molecular Plant Pathology 5:405-416.

Matsumoto M (2005) Analysis of whole-cellular fatty acids and 
anastomosis relationships of binucleate Rhizoctonia spp. from strawberry roots. Phytopathology 78:379-384.

Naito S (1996) Basidiospore dispersal and survival. In: Sneh B, Jabaji-Hare S, Neate S, Dijst G (Eds.) Rhizoctonia especies: Taxonomy, molecular, biology, ecology, pathology, and disease control. Dordrecht The Netherlands. Kluwer Academic Publishers. pp. 197-205.

Okubara PA, Schroeder KL, Paulitz TC (2008) Identification and quantification of Rhizoctonia solani and $R$. oryzae using real-time polymerase chain reaction. Techniques 98:837-847.

Pannecoucque J, Höfte M (2009) Detection of rDNA ITS polymorphism in Rhizoctonia solani AG 2-1 isolates. Mycologia 101:26-33.

Pascual CB, Toda T, Raymondo AD, Hyakumachi M (2000) Characterization by conventional techniques and PCR of Rhizoctonia solani isolates causing banded leaf sheath blight in maize. Plant Pathology 49:108-118.

Polanco T, Rodríguez R, Beaver J (1996) Variabilidad entre aislados de Rhizoctonia solani en Puerto Rico. Journal of Agriculture of the University of Puerto Rico 80:195-197.

Ronquist F, Teslenko M, Van Der Mark P, Ayres D, Darling A, Hohna S, Larget B, Liu L, Suchard M, Huelsenbeck J (2012) MRBAYES 3.2: Efficient Bayesian phylogenetic inference and model selection across a large model space. Systematics Biology 61:1-4.

Schneider JHM, Salazar O, Rubio V, Keijer J (1997) Identification of Rhizoctonia solani associated with field-grown tulips using ITS rDNA polymorphism and pectic zymograms. European Journal of Plant Pathology 103:607-622.

Van Schoonhoven A, Pastor-Corrales A (1987) Sistema estándar para la evaluación de germoplasma de fríjol. Cali Colombia. CIAT.
Sharon M, Kuninaga S, Hyakumachi M, Naito S, Sneh B (2008) Classification of Rhizoctonia spp. using rDNA-ITS sequence analysis supports the genetic basis of the classical anastomosis grouping. Mycoscience 49:93-114.

Solanki MK, Singh RK, Srivastava S, Kumar S, Kashyap PL, Srivastava AK, Arora DK (2013) Isolation and characterization of siderophore producing antagonistic rhizobacteria against Rhizoctonia solani. Journal of Basic Microbiology e10.1002.

Steadman JR, Powers K, Higgins B (1997) Screening common bean for white mold resistance using detached leaves. Annual Report of the Bean Improvement Cooperative 40:140-141.

Tamura K, Peterson D, Peterson N, Stecher G, Nei M, Kumar S (2011) MEGA5: Molecular evolutionary genetics analysis using Maximum Likelihood, Evolutionary Distance and Maximum Parsimony methods. Molecular Biology and Evolution 28:2731-2739.

White TJ, Bruns T, Lee S, Taylor J (1990) Amplification and direct sequencing of fungal ribosomal RNA genes for phylogenetics. In: Innis MA, Gelfand DH, Sninsky JJ, White TJ (Eds.) PCR protocols: A guide to methods and applications. San Diego CA, USA. Academic Press. pp. 315-322.

Wibberg D, Jelonek L, Rupp O, Kröber M, Eikmeyer FG, Goesmann A, Hartmann A, Borriss R, Grosch R, Pühler A, Schlüter A (2012) Establishment and interpretation of genome sequence of the phytopatogenic fungus Rhizoctonia solani AG 1-IB isolate 7/3/14. Journal of Biotechnology 2:142-155.

Zheng A, Lin R, Zhang D, Qin P, Xu L, Ail P, Ding L, Wang Y, Chen Y, Liu Y, Sun Z, Feng H, Liang X, Fu R, Tang C, Li Q, Zhang J, Xie Z, Deng Q, Li S, Wang S, Zhu J, Wang L, Liu H, Li P (2013) The evolution and pathogenic mechanisms of the rice sheath blight pathogen. Nature Communications 4:1424. 